\title{
Image Compression based on 4 Level AMBTC
}

\author{
Surjeet Singh Katre \\ (M.tech Student in Micro Electronics and VLSI Design) \\ Sagar Institute of Research \& Technology, RGPV University, \\ Bhopal, Madhya Pradesh, India
}

\begin{abstract}
Image compression is essential for different applications such as transmission and storage in data bases. Two methods use for image compression- lossy and lossless. In this paper we discuss about the image compression and propose a new method of lossy image compression. Its principle is based on absolute moment block truncation coding (AMBTC). In BTC, there is different method that is improving the PSNR and reduces the MSE.
\end{abstract}

\section{Keywords}

Image compression, BTC, AMBTC, PSNR, MSE

\section{INTRODUCTION}

Image compression is used for compress digital images. Our objective is to reduce redundancy of the image data in order to be able to store or transmit data in an efficient form.

Uncompressed multimedia (graphics, audio and video) data requires considerable storage capacity and transmission bandwidth. Therefore it require to reduce bits per pixel.. The recent growth of data intensive multimedia-based web applications have not only sustained the need for more efficient ways to encode signals and images but have made compression of such signals central to storage and communication technology.

The principle behind the image compression is, a common characteristic of most images is that the neighboring pixels are correlated and therefore contain redundant information. The foremost task then is to find less correlated representation of the image. Two fundamental Components of compression are redundancy and irrelevancy reduction. Redundancy reduction aims at removing duplication from the signal source (image/video). Irrelevancy reduction omits parts of the signal that will not be noticed by the signal receiver, namely the Human Visual System (HVS). In general, three types of redundancy can be identified: Coding Redundancy, Spatial Redundancy and Irrelevant Information.

\subsection{Performance Parameters}

There are two performance parameters are used to measure the performance of the image compression algorithms. One is PSNR (peak signal to noise ratio) and second is Mean square error (MSE). PSNR is the measurement of the peak error between the compressed image and original image. The higher the PSNR contains better quality of image. The two quantitative measures are defined as

$$
\begin{aligned}
& \text { MSE }=\frac{1}{\mathrm{~N}} \sum_{\mathrm{i}=1}^{\mathrm{N}}\left(\mathrm{y}_{\mathrm{i}}-\mathrm{x}_{\mathrm{i}}\right)^{2} \\
& \mathrm{SNR}=-10 \cdot \log _{10}\left[\frac{\mathrm{MSE}}{225^{2}}\right]
\end{aligned}
$$

BTC has been used for compressing digital monochrome images. BTC for monochrome image compression was introduced by Delp and Mitchell [1]. BTC has the advantage of being easy to implement compared to vector quantization and transform coding. In the BTC method at first calculate the block mean and standard deviation. In the encoding procedure of BTC the image is first partitioned in to a set of non overlapping blocks, and then the first two statistical moments and the bit plane are computed. At decoder, each of the encoded image block are reconstructed using the bit plane and the two statistical moments. It achieves 2 bits per pixel (bpp) with low computational complexity.

\section{RELATED WORKS}

A simple and fast variant of BTC called Absolute Moment Block Truncation Coding (AMBTC) is presented by Lema and Mitchell [3]. It preserves the higher mean and lower mean of the blocks. However the bit rate achieved is 2 bpp which is same as in the original BTC. In order to reduce the bit rate many techniques have been used to code the statistical moments and the bit plane of BTC. To reduce bpp many modification of BTC have been proposed. Udipikar and Raina [5] give BTC image compression using Vector Quantization (VQ) and the bit rate in the range of $1.0-1.5 \mathrm{bpp}$ is achieved. Zeng and Neuvo [8] have proposed two BTC methods with VQ schemes. The hybrid BTC / VQ techniques reduce the bit rate, but they require more computation for encoding the code book generation. Yung-Gi Wu [6] proposed probability based block truncation image bit plane coding. YuChen $\mathrm{Hu}$ [7] presented a modified BTC with predictive technique and bit plane coding with edge pattern. Hence the improvements on BTC are for improve PSNR and to reduce bpp and low computational complexity.

\section{IMPORTANCE OF THE STUDY PROBLEM STATEMENT}

In this paper we propose an image compression scheme based on Multilevel AMBTC. From the experimental results, we found that the proposed scheme gives good image quality with low bit rate.

In Section II a review of the AMBTC method is given. Section III describes the proposed image compression scheme.

The experimental results are discussed in Section IV. Finally we conclude this paper in Section V.

\section{ABSOLUTE MOMENT BLOCK TRUNCATION CODING}

This compression technique reduces the computational complexity and achieves the optimum minimum mean square error and PSNR [3]. In this work we have compressed the image using AMBTC algorithm. The following steps are used:-

1) Original image is segmented into blocks of size $4 \times 4$ or $8 \times 8$ for processing.

2) Find the mean of each block also the mean of the higher range and lower range. 


$$
\begin{array}{ll}
\text { mean } & \overline{\mathrm{x}}=\frac{1}{\mathrm{~m}} \cdot \sum_{\mathrm{i}=1}^{\mathrm{m}} \mathrm{x}_{\mathrm{i}} \\
\text { Low mean } & \overline{\mathrm{x}_{\mathrm{l}}}=\frac{1}{\mathrm{~m}-\mathrm{q}} \sum_{\mathrm{xi} \leq \overline{\mathrm{x}}}^{\mathrm{m}} \mathrm{x}_{\mathrm{i}} \\
\text { High mean } & \overline{\mathrm{x}_{\mathrm{h}}}=\frac{1}{\mathrm{q}} \sum_{\mathrm{x} \mathrm{i} \geq-\overline{\mathrm{x}}}^{\mathrm{xi}}
\end{array}
$$

Where $\mathrm{q}$ is the number of pixels whose gray level is greater than $\overline{\mathrm{x}}$.

A binary block, denoted by $\mathrm{b}$, is also used to represent the pixels. We can use "1" to represent a pixel whose gray level is greater than or equal to $\overline{\mathrm{x}}$ and " 0 " to represent a pixel whose gray level is less than $\overline{\mathrm{x}}$. Assume that we use 8 bits to represent $\overline{\mathrm{x}_{\mathrm{h}}}, \overline{\mathrm{x}_{\mathrm{l}}}$ respectively. Then the total number of bits required for a block is $8+8+16=32$ bits. Thus, the bit rate for the AMBTC algorithm is 2 bpp . In the decoder, an image block is reconstructed by replacing the 1 's with $\overline{\mathrm{x}_{\mathrm{h}}}$ and the 0 's by $\overline{\mathrm{x}_{\mathrm{l}}}$. In the AMBTC, we need 16 bits to code the bit plane which is same as in the BTC. But, AMBTC requires less computation than BTC.

\section{PROPOSED SCHEME}

In the proposed compression scheme makes use of AMBTC.

\section{Encoding Steps:}

The input image is divided into blocks of size $4 \times 4$ pixels. For each block, the high mean and the low mean, called the quantizers are computed using the equations (2) and (3) as in AMBTC. While encoding, to generate four quantizers, the step value is computed using the equation (4)

$$
\mathrm{SV}=\frac{\left(\overline{\mathrm{x}}_{\mathrm{h}}-\overline{\mathrm{x}}_{1}\right)}{3}
$$

The four quantizers, other than the $\bar{x}_{h}$ and $\bar{x}_{l}$ are computed using the equations (5) thru.(8).

$$
\begin{aligned}
& \mathrm{Q}_{1}=\overline{\mathrm{x}}_{\mathrm{l}} \\
& \mathrm{Q}_{2}=\overline{\mathrm{x}}_{\mathrm{l}}+\mathrm{sv} \\
& \mathrm{Q}_{3}=\mathrm{Q}_{2}+\mathrm{sv} \\
& \mathrm{Q}_{4}=\overline{\mathrm{x}}_{\mathrm{h}}
\end{aligned}
$$

For each block, the bit plane is generated as follows:

If the pixel value is closer to $Q_{1}$, it is coded as 00 . If the pixel value is closer to $Q_{2}$, it is coded as 01 , If closer to $Q_{3}$, it is coded as 10 and If closer to $\mathrm{Q}_{4}$, it is coded as 11 .

There is bit plane of size 32 bits is generated rather than 16 bits. Here only the two quantizers $\mathrm{Q}_{1}$ and $\mathrm{Q}_{4}$ are preserved with the bit plane. The quantizers $\mathrm{Q}_{2}$ and $\mathrm{Q}_{3}$ are consider only when encoding and decoding take place and not consider with the bit plane. There are four different levels for bpp reducing:

In $1^{\text {st }}$ level, for each input image block, a bit plane of size 32 bits along with the quantizers $\mathrm{Q}_{1}$ and $\mathrm{Q}_{4}$ of size 16 bits are stored therefore bit rate of $3 \mathrm{bpp}$.

In the second level of compression, the $2^{\text {nd }}, 6^{\text {th }}, 10^{\text {th }}$ and the $14^{\text {th }}$ elements of the bit plane are dropped, to reduce the redundancy of image.

The above set $\{2,6,10,14\}$ of pixels can be dropped because they have pixels both in the left and right sides. The bpp obtained out of dropping the above mentioned elements of the bit plane is reduced to $2.5 \mathrm{bpp}$ with only less degradation in the quality.
In the $3^{\text {rd }}$ level of reducing the bit rate further, the boldfaced elements as given in Fig. 1 are dropped. At the encoding stage, the dropped bits are regenerated using the equation set (9). As a result, the bpp is reduced to 2 with better PSNR when compared to AMBTC.

$$
\left(\begin{array}{lcccc}
1 & 2 & 3 & & \mathbf{4} \\
5 & \mathbf{6} & 7 & & 8 \\
9 & \mathbf{1 0} & \mathbf{1 1} & & 12 \\
\mathbf{1 3} & & 14 & 15 & \mathbf{1 6}
\end{array}\right)
$$

\section{Fig1: Position of element to be dropped}

$$
\left\{\begin{array}{ll}
x_{i}=\frac{1}{2}\left(x_{i+1}+x_{i+4}\right) & \text { for } i=1,11 \\
x_{i}=\frac{1}{2}\left(x_{i-1}+x_{i+4}\right) & \text { for } i=4,10 \\
x_{i}=\frac{1}{2}\left(x_{i-1}+x_{i-4}\right) & \text { for } i=6,16 \\
x_{i}=\frac{1}{2}\left(x_{i+1}+x_{i-4}\right) & \text { for } i=7,13
\end{array}\right\}
$$

In the 4th level of compression, the statistical moments Q1 and Q4 are divided by 4 to reduce the number of bits required to represent them.

\section{Decoding Steps:}

1. Input the Bit plane and the two quantizers: $\mathrm{Q}_{1}$ and $\mathrm{Q}_{4}$.

2. Compute the four quantizing levels using the equations through (5) thru. (8).

3. Reconstruction of compressed image is done in three levels as follows:

Level1: Reconstruct the image as decoding the elements of the bit plane as follows:

i. if the element is 00 , code it as Q1

ii. if 01, code it as Q2

iii. if 10, code it as Q3

iv. if 11 , code it as Q4

or

Level2: Recompute the dropped elements of the bit plane as follows:

$>3$ average of $2 \& 4$

$>7$ average of $3,6 \& 8$

$>11$ average of $10,7 \& 12$

$>15$ average of $14,11 \& 16$

And perform Level2 actions

or

Level3: dropped elements are Re-compute using the equation set (9) and perform Level 2 actions.

or

Level4: Compute $\mathrm{Q}_{1}=\mathrm{Q}_{1} * 4$ and $\mathrm{Q}_{4}=\mathrm{Q}_{4} * 4$ along with Level3 computations and perform Level2 actions. 


\section{RESULT}

Standard images Cameraman, Boats, Bridge, Baboon and Lena of size $256 \times 256$ pixels are taken for experiment. The input images taken for the study are given in Fig. 2.

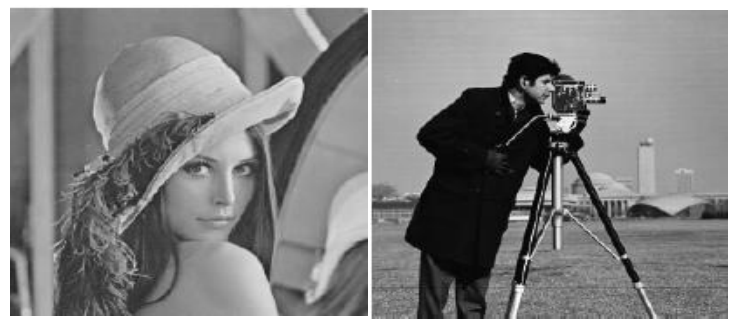

(a) Lena

(b) cameraman

Fig2. Input Image taken for study
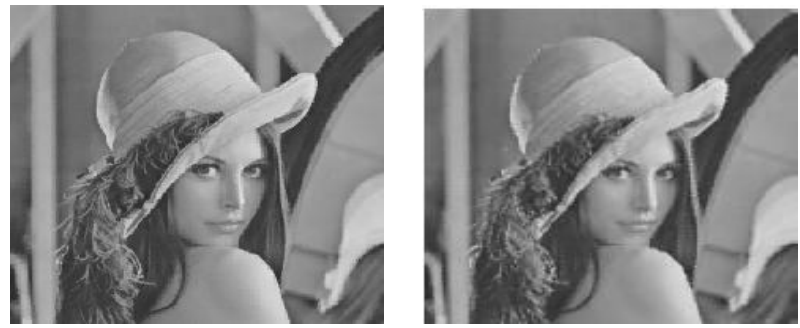

AMBTC

Multilevel AMBTC

(PSNR 34.90, bpp-2)

(PSNR-34.93, bpp-1.75)

Fig3: Decoded Image by AMBTC and Multilevel AMBTC

The PSNR and the bpp obtained with the existing AMBTC Method and the proposed method is shown in Table I. The AMBTC method yields a bit-rate of $2 \mathrm{bpp}$. The average PSNR obtained is 33.53.But in our method bpp is 1.75 and PSNR is 35.60 .

Table1: The PSNR and the bpp in AMBTC Method and the proposed methods

\begin{tabular}{|l|l|l|l|l|}
\hline \multirow{2}{*}{ Image } & \multicolumn{2}{|l|}{ AMBTC } & \multicolumn{2}{l|}{$\begin{array}{l}\text { Proposed } \\
\text { Method(level4) }\end{array}$} \\
\cline { 2 - 5 } & PSNR & Bpp & PSNR & bpp \\
\hline Lena & 34.90 & 2 & 35.92 & 1.75 \\
\hline Cameraman & 32.17 & 2 & 35.28 & 1.75 \\
\hline Average & 33.53 & 2 & 35.60 & 1.75 \\
\hline
\end{tabular}

\section{CONCLUSION}

The compression algorithm depends on the three factors: quality of image, amount of compression and speed of compression. When compared to conventional AMBTC method, the bit-rate is reduced on an average from 2.00 to $1.75 \mathrm{bpp}$ and the PSNR is also raised to 35.60 in our method. Our method can also be applied to color images as well as the gray scale images. In future if we increase the level of quantization we can increase the PSNR at better level.

\section{REFERENCE}

[1] E.J. Delp, O.R. Mitchell, "Image Compression using Block Truncation Coding", IEEE, Trans. Communications, Vol. 27, pp.1335-1342, September 1979.

[2] Jain A.K., "Image Data Compression: A Review", IEEE, pp. 349-380, 1981.

[3] M.D.Lema and O.R.Mitchell, "Absolute Moment Block Truncation Coding and its Application to Color images," IEEE Trans. On Communications, Vol. 32, pp. 11481157,1984

[4] N.M. Nasrabadi, R.B. King, "Image coding using vector quantization: a review," IEEE Transactions on Communications COM-36, pp. 957-971, 1998

[5] Udpikar V., Raina J. (1987) "BTC Image Coding Using Vector Quantization." IEEE Transactions on communications, 35, 352-356.

[6] Yung-Gi Wu and Shen-Chuan Tai, "An efficient BTC image compression technique," (pp.-pp. 317-324) IEEE Transaction paper, 1998

[7] $\mathrm{Yu}-\mathrm{Chen} \mathrm{Hu}$, "Predictive moment preserving block truncation coding for gray level image compression," Journal of electroninc Imaging, Vol.13(4), pp. 871-877, 2004

[8] Zeng B., Neuvo Y. "Interpolative BTC Image Coding with Vector Quantization." IEEE Transactions on Communications, 41, 1436-1438, 1993.

[9] K. Somasundaram et al. "RGB \& gray scale component on MPQ-BTC in image compression" International Journal on Computer Science and Engineering (IJCSE) issn : 0975-3397 Vol. 3 No. 4 Apr 2011 